\title{
Paradox of Contact Angle Selection on Stretched Soft Solids
}

\author{
Jacco H. Snoeijer, ${ }^{1,2}$ Etienne Rolley, ${ }^{3}$ and Bruno Andreotti ${ }^{3}$ \\ ${ }^{1}$ Physics of Fluids Group, Faculty of Science and Technology, University of Twente, P.O. Box 217, 7500 AE Enschede, Netherlands \\ ${ }^{2}$ Department of Applied Physics, Eindhoven University of Technology, P.O. Box 513, 5600 MB, Eindhoven, Netherlands \\ ${ }^{3}$ Laboratoire de Physique Statistique, UMR 8550 ENS-CNRS, Univ. Paris-Diderot, 24 rue Lhomond, 75005, Paris, France
}

(Received 12 March 2018; published 8 August 2018)

\begin{abstract}
The interfacial mechanics of soft elastic networks plays a central role in biological and technological contexts. Yet, effects of solid capillarity have remained controversial, primarily due to the strain-dependent surface energy. Here we derive the equations that govern the selection of contact angles of liquid drops on elastic surfaces from variational principles. It is found that the substrate's elasticity imposes a nontrivial condition that relates pinning, hysteresis, and contact line mobility to the so-called Shuttleworth effect. We experimentally validate our theory for droplets on a silicone gel, revealing an enhanced contact line mobility when stretching the substrate.
\end{abstract}

DOI: 10.1103/PhysRevLett.121.068003

The functionality of extremely soft materials emerges from a combination of bulk elasticity and surface effects [1-5]. However, the interfacial mechanics of soft solids, typically reticulated polymer networks, is notoriously difficult to probe experimentally [6,7]. A very promising route to quantitatively measure solid surface tension is via the contact angles of liquid drops [8-10]. The wetting on soft solids is intermediate between the case of rigid solids for which the contact angle is selected by Young's law, and the case of a liquid-liquid interface for which contact angles are selected by Neumann's law [11-15]. However, the interpretation of contact angles on stretched solids has recently raised a controversy, with similar experiments leading opposite conclusions on the coupling between elasticity and surface tension $[9,10]$. In this Letter, we resolve this paradox by deriving the equilibrium conditions at the contact line from first principles. We reveal a previously ignored condition that must be satisfied to avoid pinning and contact angle hysteresis. Predictions are validated by dynamical experiments [16-20], elucidating how stretching the substrate affects hysteresis and contact line mobility.

The challenge arises due to the fundamental difference between the capillarity of solids and liquids. For solid interfaces, the excess energy $\gamma$ per unit area generically depends on the surface strain $\epsilon$. Contrarily to liquid interfaces, the surface tension $\Upsilon$ in the interface therefore differs from $\gamma$ owing to the "Shuttleworth effect": surface energy and surface tension are related by the Shuttleworth equation $[3,4,21]$

$$
\Upsilon(\epsilon)=\frac{d}{d \epsilon}[(1+\epsilon) \gamma(\epsilon)]=\gamma+(1+\epsilon) \gamma^{\prime} .
$$

Since we do not wish to restrict the analysis to small strain, we here retain the factor $(1+\epsilon)$ on the right. The first evidence of a strong coupling between surface energy and strain - a strong Shuttleworth effect-for reticulated polymers was obtained using an elastic (polyvinylsiloxane) Wilhelmy plate, which allows for the measurement of the difference $\gamma_{\mathrm{SL}}^{\prime}-\gamma_{\mathrm{SV}}^{\prime}$ between solid-liquid and solid-vapor interfaces [3,22]. Recent studies addressed the Shuttleworth effect through contact angles of liquid drops (cf. Fig. 1), in particular their variation when stretching the substrate $[9,10]$. Intriguingly, the observations led to contradictory interpretations. Xu et al. [9] observed that stretching a silicone gel leads to a significant increase of the solid angle $\theta_{S}$, which was attributed to a strong Shuttleworth effect, i.e., to a large value of $\gamma^{\prime} / \gamma$. Schulman et al. [10], by contrast, conclude that there is no Shuttleworth effect $\left(\gamma^{\prime}=0\right)$ for a broad range of different elastomers. This is based on the striking absence of any dependence on

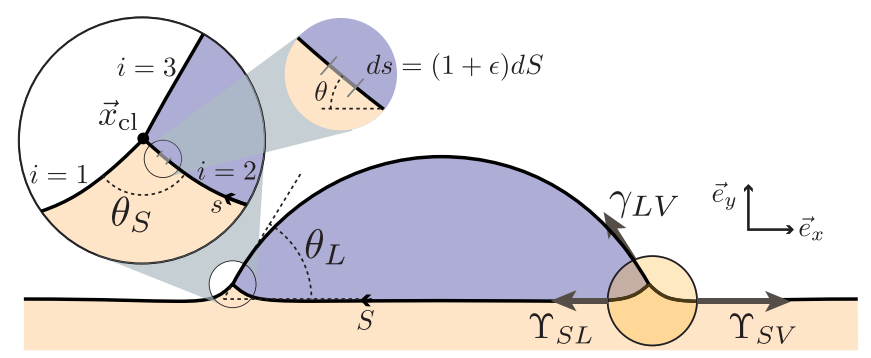

FIG. 1. Contact angles on a soft solid: The liquid angle $\theta_{L}$ of the drop (main panel) and the solid angle $\theta_{S}$ of the wetting ridge (zoom). In the presented theory, the three interfaces $(i=1,2,3)$ are parametrized using curvilinear coordinates $s$, related to the reference coordinate $S$ by the strain $\epsilon_{i}(S)$, while the local angle of the interface is $\theta_{i}(\mathrm{~S})$. The interfaces meet at the contact line position $\vec{x}_{\mathrm{cl}}$. The effect of surface tensions $\Upsilon_{\mathrm{SV}}$ and $\Upsilon_{\mathrm{SL}}$ is illustrated by a force balance on the circular zone near the contact line on the right [Eqs. (7), (8)]. 
strain of the liquid angle $\theta_{L}$ : While the contact angle on (stiff) glassy polymers varies with the external strain, no variation of $\theta_{L}$ was found for (soft) elastomers up to $100 \%$ strain.

Exact wetting conditions from variational analysis.Here we set out to derive the complete set of equilibrium conditions for soft wetting, by minimizing the sum of elastic and capillary energies. The geometry is sketched in Fig. 1, showing the three interfaces near the contact line. The index $i=1$ refers to the solid-vapor interface, $i=2$ refers to the solid-liquid interface, and $i=3$ refers to the liquid-vapor interface. From the outset we consider that the size of elastic deformation $\sim \gamma / G$ is large compared to molecular scales ( $G$ being the static shear modulus), so that a wetting ridge develops and the interfaces are sharp. The free energy per unit width reads

$$
\mathcal{F}=\mathcal{F}_{e}+\sum_{i} \int_{-\infty}^{r_{i}} \gamma_{i}\left(\epsilon_{i}\right) d s
$$

where $\mathcal{F}_{e}$ is the elastic energy, and $s$ is the curvilinear coordinate along each of the interfaces. The integrals run from a position far from the contact line ("- $\infty$ ") to the contact line $\left(s=r_{i}\right)$. The strain field $\epsilon_{i}$ is defined for the elastic interfaces $(i=1,2)$ and is actually a function of the reference coordinate $S$. This coordinate refers to a material point at the interface in its undeformed state prior to the deposition of the liquid drop. The geometric connection between the deformed state and reference state (Fig. 1) reads $d s=\left(1+\epsilon_{i}\right) d S$. Hence, the variation of surface energy gives $\delta\left[\gamma_{i}\left(\epsilon_{i}\right) d s\right]=\Upsilon \delta \epsilon_{i}(S) d S$, with $\Upsilon_{i}$ as defined in Eq. (1). The interfaces are fully specified when complementing the strain with the local angle $\theta_{i}(S)$, which must also be varied to obtain the equilibrium. Minimization of $\mathcal{F}$ then implies $\delta \mathcal{F}_{e} / \delta \epsilon_{i}+\Upsilon_{i}=0$ and $\delta \mathcal{F}_{e} / \delta \theta_{i}=0$.

It is convenient to express the derivatives of the elastic energy in terms of the elastic traction $\vec{\sigma}$, defined as the elastic force per unit (deformed) area. It can be inferred from the variation of interface displacements $\delta \vec{u}$, as derived in the Supplemental Material [23]. From the kinematic connection between $\delta \epsilon, \delta \theta$, and $\delta \vec{u}$, one finds (omitting the indices $i=1,2$ )

$$
\vec{\sigma}(s)=\frac{\delta \mathcal{F}_{e}}{\delta \vec{u}(s)}=-\frac{d}{d s}\left(\frac{\delta \mathcal{F}_{e}}{\delta \epsilon(S)} \vec{t}+\frac{1}{1+\epsilon} \frac{\delta \mathcal{F}_{e}}{\delta \theta(S)} \vec{n}\right) .
$$

Here $\vec{t}$ and $\vec{n}$, respectively, are tangential and normal unit vectors along the interfaces. Combined with the mentioned conditions for $\delta \mathcal{F}=0$, the traction (3) becomes

$$
\vec{\sigma}(s)=\frac{d(\Upsilon \vec{t})}{d s}=\Upsilon \frac{d \theta}{d s} \vec{n}+\frac{d \Upsilon}{d s} \vec{t}
$$

The elastic traction $\vec{\sigma}$ balances the normal Laplace pressure due to curvature, as in liquid capillarity and tangential

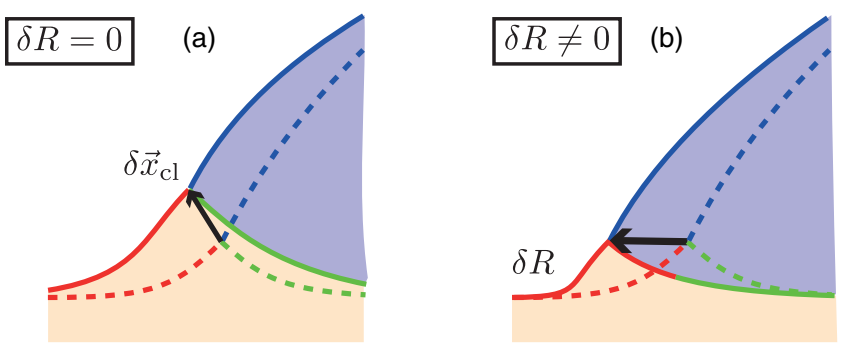

FIG. 2. Equilibrium requires that the energy is stationary with respect to any possible mode of contact line displacement, as sketched in panels (a) and (b). (a) Displacement mode at fixed material point $(\delta R=0)$, where the molecules from each phase joining at the contact line are moved together, gives rise to the Neumann condition (5). (b) Displacement mode with variable material point $(\delta R \neq 0)$ gives rise to a no-pinning condition (6). Color indicates whether material points prior to displacement belonged to the wet part (green) or dry part (red) of the solid. At equilibrium, both Eqs. (5) and (6) must be satisfied.

Marangoni stress due to gradients of surface tension, originating here from inhomogeneities of $\epsilon(S)$.

The key purpose of the analysis, however, is to derive the boundary conditions at the contact line. For this we need to specify how the three interfaces $i=1,2,3$ are connected at their respective end points at $S=R_{i}$. Obviously, the interfaces meet at a common position $\vec{x}_{\mathrm{cl}}$ (Fig. 1), which must be imposed as a constraint. The constraint does not affect Eq. (4), but is crucial for the boundary conditions that are obtained by virtual displacements of the contact line. A first mode of displacement consists of varying the contact line position $\delta \vec{x}_{\mathrm{cl}}$ while keeping the material coordinates at the contact line fixed $\left[\delta R_{i}=0\right.$, see Fig. 2(a)]. This gives a boundary condition evaluated at the contact line (Supplemental Material [23]):

$$
\sum \Upsilon_{i} \vec{t}_{i}=\overrightarrow{0}
$$

This is the Neumann law that determines $\theta_{S}$, commonly used but here derived from variational principles. Importantly, Eq. (5) is obtained by a variation of the contact line position while keeping $S=R_{1}$ and $S=R_{2}$ constant; i.e., the threephase system surrounding the contact line is displaced as a whole, without relative motion [Fig. 2(a)]. The free energy, however, should not only be minimized when the contact line is materially displaced by $\delta \vec{x}_{\mathrm{cl}}$, but in addition when the liquid at the contact line is moved with respect to the solid. In the absence of contact line pinning (no heterogeneities of the substrate), one should therefore consider changes of the material point below the contact line. As is illustrated in Fig. 2(b), the liquid can freely move and change the solid molecules that are present at $\vec{x}_{\mathrm{cl}}$. This must be accounted for by allowing the variation $\delta R_{1}=-\delta R_{2}$, exchanging material points from the dry $(i=1)$ to the wetted interface $(i=2)$. The variation $\delta R$ gives a new boundary condition at the contact line, which will be referred to as the no-pinning condition: 


$$
\Delta\left[(1+\epsilon)^{2} \gamma^{\prime}(\epsilon)-\frac{\partial \mathcal{F}_{e}}{\partial R}\right]=0 .
$$

Here $\Delta[\cdots]$ denotes the difference between both sides of the contact line along the solid (Supplemental Material [23]). Equilibrium thus imposes two boundary conditions (5) and (6), both of which must be satisfied. Although the appearance of two conditions is logically associated with the position $\vec{x}_{\mathrm{cl}}$ and the material coordinate of the solid $R_{i}$, Eq. (6) had never been considered so far.

The new condition can be interpreted by analogy with the equality of "chemical potentials" at a liquid-vapor interface: while the chemical potential governs the equilibrium with respect to transport of material across such an interface, Eq. (6) ensures the equilibrium with respect to transport of elastic material (by $\delta R_{i}$ ) across the contact line. For a given combination of $\gamma_{\mathrm{SV}}(\epsilon)$ and $\gamma_{\mathrm{SL}}(\epsilon)$, the strain must thus adapt in order to satisfy (6). This is analogous to the adaptation of liquid and vapor densities at two-phase coexistence.

In order to compare to macroscopic experiments, we need to translate these results to regions far away from the wetting ridge (Fig. 1). To this end we first integrate Eq. (4) across the contact line, which using Eq. (5) gives

$$
\begin{gathered}
\Upsilon_{\mathrm{SL}}-\Upsilon_{\mathrm{SV}}+\gamma_{\mathrm{LV}} \cos \theta_{L}=\vec{e}_{x} \cdot \int_{-\infty}^{\infty} \vec{\sigma} d s, \\
\gamma_{\mathrm{LV}} \sin \theta_{L}=\vec{e}_{y} \cdot \int_{-\infty}^{\infty} \vec{\sigma} d s .
\end{gathered}
$$

This expresses the resultant force of the three surface tensions on the volume indicated in Fig. 1, which must be balanced by the elastic stress integrated over the contact line region. Besides the normal force, we recover the appearance of a tangential stress across the contact line $[3,22,24]$. Recombining these equations with the no-pinning condition (6) this can be expressed as

$$
\begin{aligned}
& \left(1+\epsilon_{\infty}\right)\left(\gamma_{\mathrm{SL}}-\gamma_{\mathrm{SV}}+\gamma_{\mathrm{LV}} \cos \theta_{L}\right) \\
& \quad=-\Delta\left[(1+\epsilon)^{2} \gamma^{\prime}\right]+\int_{-\infty}^{\infty} \vec{\sigma} \cdot\left[(1+\epsilon) \vec{t}-\left(1+\epsilon_{\infty}\right) \vec{e}_{x}\right] d s .
\end{aligned}
$$

This is the generalization of Young's law for the liquid angle $\theta_{L}$ far away from the contact line, also applicable when the substrate is uniaxially strained to a value $\epsilon_{\infty}$.

The paradox.-So far the derivation has been fully exact and provides the thermodynamic framework for partial wetting on soft solids, valid also for large deformations. Resolving the experimental controversy regarding the Shuttleworth effect calls for a quantitative prediction, and for this reason we evaluate these exact results in the framework of linear elasticity for which $|\epsilon| \ll 1$. The elastic energy is then obtained by the surface integral $\mathcal{F}_{e}=\int d s \frac{1}{2} \vec{u} \cdot \vec{\sigma}$. Hence, the boundary condition (6) then simplifies to $\Delta\left[\gamma^{\prime}(0)-\frac{1}{2} \vec{u} \cdot \vec{\sigma}\right]=0$. A stress discontinuity is not admitted in linear elasticity, as it would lead to a logarithmic singularity of slope. Hence, we deduce that the newly found no-pinning condition (6) enforces continuity of $\gamma^{\prime}(0)$, so that $\gamma_{\mathrm{SV}}^{\prime}=\gamma_{\mathrm{SL}}^{\prime}$. Far from the contact line this implies that Eq. (9) reduces to the standard Young equation $\gamma_{\mathrm{SL}}-\gamma_{\mathrm{SV}}+\gamma_{\mathrm{LV}} \cos \theta_{L}=0$. All the above remains valid for large $\epsilon_{\infty}$, as long as the perturbation induced by the deposited drop remains in the regime of linear response.

This explains why $\theta_{L}$ must remain constant on stretched soft solids [10], even when the Shuttleworth effect leads to a change of $\theta_{S}$, an experimental fact that has been observed by simultaneous measurement of $\theta_{S}$ and $\theta_{L}$ in PDMS in Ref. [9]. Namely, the no-pinning condition $\gamma_{\mathrm{SV}}^{\prime}=\gamma_{\mathrm{SL}}^{\prime}$ imposes that the difference $\gamma_{\mathrm{SV}}-\gamma_{\mathrm{SL}}$ stays constant. Hence, as long as the drop's distortion of the solid is within linear response, the constancy of $\theta_{L}$ is a direct consequence of the lack of hysteresis, and as such $\theta_{L}$ cannot predict the absence or presence of the Shuttleworth effect on soft elastic solids. The situation is different for glassy polymers, which are much stiffer and for which changes of $\theta_{L}$ have been observed when stretching the substrate [10]. In that case the associated deformations $\sim \gamma / G$ are subnanometric so that the solid remains essentially flat. In our macroscopic framework, this corresponds to the rigid limit in which the droplet does not induce any elastic deformation-hence, the strain remains everywhere fixed at the experimentally imposed value $\epsilon_{\infty}$. The variation of the horizontal contact line position is then naturally connected to a variation of the material point according to $\delta x_{\mathrm{cl}}=\left(1+\epsilon_{\infty}\right) \delta R$ so that the no-pinning condition (due to $\delta R$ ) is the same as Young's equation (due to $\delta x_{\mathrm{cl}}$ in the rigid limit). So indeed, for stiff polymers there is no thermodynamic reason why $\theta_{L}$ should remain constant when the externally imposed strain $\epsilon_{\infty}$ is varied experimentally.

Turning back to the case of soft reticulated polymer networks, for which $\gamma / G$ is significantly larger than the molecular scale, the equality $\gamma_{\mathrm{SV}}^{\prime}=\gamma_{\mathrm{SL}}^{\prime}$ at small strain is not expected to be universally obeyed. This will depend on the relevant constitutive relations $\gamma(\epsilon)$, which is a topic that is currently beginning to be explored $[25,26]$. One could expect the emergence of large strain at the contact line to satisfy the no-pinning condition (6). If such an equilibrium of chemical potential is not compatible with the constitutive relations, pinning must be observed. Indeed, elastomeric surfaces can exhibit a strong contact angle hysteresis, which we suggest finds its origin in the Shuttleworth effect. For example, the Wilhelmy plate experiment of Ref. [22] for which strong hysteresis was present, exhibited $\gamma_{\mathrm{SL}}^{\prime} \neq \gamma_{\mathrm{SV}}^{\prime}$ as inferred from the tangential stress balance (7).

Contact angle selection from dynamic spreading.-We now experimentally validate this interpretation framework: linear response and absence of hysteresis necessarily imply 

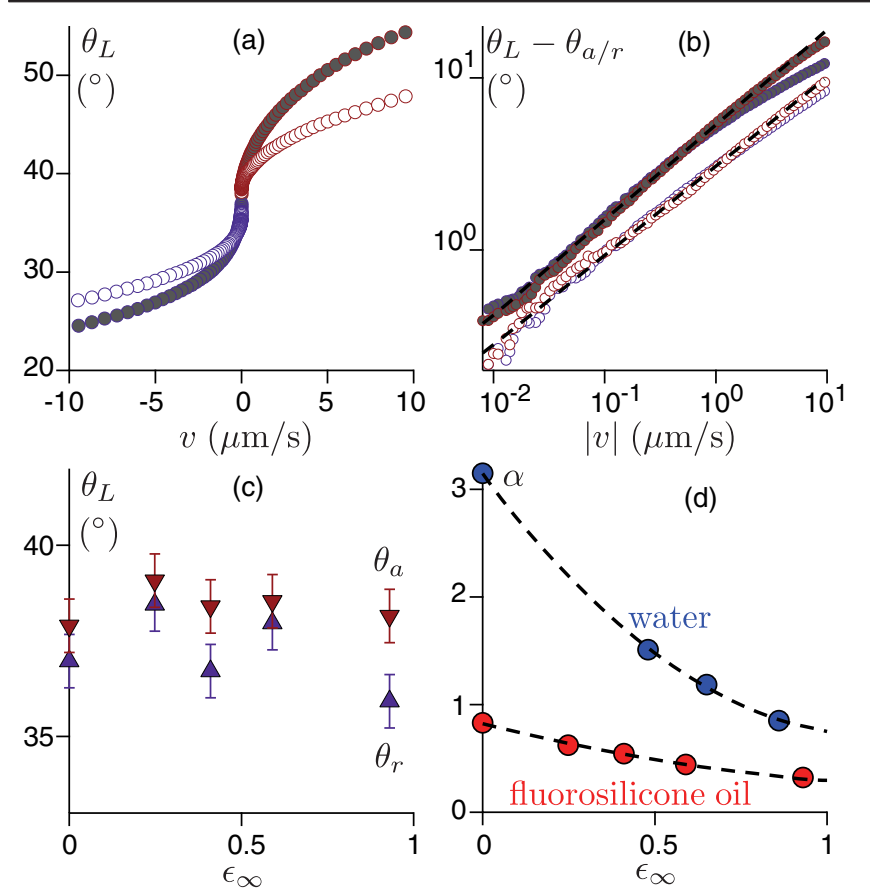

FIG. 3. Pinning and enhanced contact line mobility. (a) Liquidvapor macroscopic contact angle $\theta_{L}$ with respect to the undeformed solid surface as a function of the contact line velocity $v$ (liquid: fluorosilicone oil). Closed and open symbols: unstrained and strained $\left(\epsilon_{\infty}=0.93\right)$ samples respectively. (b) Contact angle rotations $\theta_{L}-\theta_{a / r}$ vary like $v^{n}$, with $n$ obtained from loss modulus measurement as expected from (10) $(n=0.55$ for $\epsilon_{\infty}=0$ and $n=0.50$ for $\epsilon_{\infty}=0.93$ ). (c) Liquid contact angles $\theta_{a / r}$ in the limit $v \rightarrow 0^{+} / 0^{-}$, as a function of the applied strain $\epsilon_{\infty}$. (d) Contact line friction factor $\alpha$ defined by Eq. (10) as a function of strain $\epsilon_{\infty}$.

the equality $\gamma_{\mathrm{SV}}^{\prime}=\gamma_{\mathrm{SL}}^{\prime}$-and hence $\theta_{L}$ independent of strain. Since hysteresis plays a key role in our theory, we set out to perform a proper quantification of hysteresis. This calls for dynamic spreading experiments, where the contact angle is measured versus contact line velocity $v$ [27]. The hysteresis is then inferred from the limit of vanishing velocity, comparing the advancing motion $\left(v \rightarrow 0^{+}\right)$and the receding motion $\left(v \rightarrow 0^{-}\right)$. Here we use the same set up as in Ref. [20] to measure the macroscopic contact angle $\theta_{L}$, adapted to impose a uniaxial strain $\epsilon_{\infty}$ to a $6 \mathrm{~mm}$ thick PDMS gel (Dow Corning CY52-276, prepared in 1:1 ratio) molded in a Petri dish. After curing at room temperature during $24 \mathrm{~h}, 20 \mathrm{~mm}$-wide strips are cut, stretched, and clamped on a rigid plate. A liquid droplet is inflated or deflated with a syringe to impose an advancing or a receding motion, measured along the stretching direction. Milli-Q water and fluorosilicone oil (poly(3,3,3-trifluoropropylmethylsiloxane), Gelest FMS 121) were used.

The resulting liquid contact angle $\theta_{L}$ is shown in Fig. 3(a) as a function of the contact line velocity $v$, for unstrained (closed circles) and strained samples (open circles). The curves are apparently continuous at $v=0$, pointing to a nearly perfect absence of hysteresis. Both the advancing and receding motions exhibit a power-law dependence $\theta_{L}-\theta_{a / r} \sim|v|^{n}$ over more than two decades in velocity [Fig. 3(b)]. This allows us to determine accurately the advancing $\theta_{a}$ and receding $\theta_{r}$ angles, revealing a small $\left(\sim 1^{\circ}\right)$ constant hysteresis [Fig. 3(c)]. Furthermore, the equilibrium liquid angle $\theta_{a} \simeq \theta_{r}$ is independent of the imposed strain $\epsilon_{\infty}$, consistently with previous independent experiments $[9,10]$. The condition $\gamma_{\mathrm{SL}}^{\prime}=\gamma_{\mathrm{SV}}^{\prime}$, predicted by our theory in the absence of hysteresis, is therefore fulfilled in our experiment.

Another remarkable result of Fig. 3 is that the spreading velocities are strongly enhanced upon stretching the solid. Namely, comparing the data for strained (open) and unstrained (closed) samples for a given $\theta_{L}$, the velocity $|v|$ is larger by a factor of 3 . Hence, we observe that stretching leads to an enhanced wetting mobility. Below we demonstrate how this effect serves as a simple diagnostic for the presence of a Shuttleworth effect.

The final step is to experimentally verify our assumption of linear response and to explain the enhanced wetting mobility on a stretched gel. The gel's linear rheology under uniaxial strain turns out to only weakly depend on the imposed $\epsilon_{\infty}$ (Supplemental Material [23]). The loss modulus $G^{\prime \prime}$ depends as a power law of the angular frequency $\omega$ : $G^{\prime \prime} \propto G(\omega \tau)^{n}$, where the cross-over time $\tau$ and the exponent $n$ marginally depend on $\epsilon_{\infty}$ (Supplemental Material [23]). This enables us to use the dynamical theory from Ref. [20], relating $\theta_{L}$ to the contact line velocity $v$

$$
\theta_{L}-\theta_{a / r}=\alpha\left(\frac{G|v| \tau}{\gamma_{\mathrm{LV}} \sin \theta_{L}}\right)^{n}
$$

where the dimensionless friction factor $\alpha$ depends on the geometry of the ridge. Indeed, the exponents $n$ measured for $\theta_{L}$ and in the linear rheological measurement are found to be consistent, as predicted by Eq. (10). The agreement of these exponents provides direct proof that the droplet dynamics probes the substrate within linear response, even when the prestrain $\epsilon_{\infty}$ is not small.

The experimentally measured friction factor $\alpha$ defined by Eq. (10) is reported as a function of $\epsilon_{\infty}$ in Fig. 3(d). The reduction of friction with strain can be attributed to the Shuttleworth effect, via a gradual increase of $\theta_{S}$ : A shallower wetting ridge leads to a smoother motion of material points and hence to less dissipation. This effect can be estimated from viscoelastic theory [20] (based on constant surface tensions), suggesting the scaling

$$
\alpha \sim \cos ^{1+n}\left(\theta_{S} / 2\right) .
$$

Hence, the reduced friction $\alpha$ in our spreading experiments [Fig. 3(d)] points to a Shuttleworth-induced gradual increase of $\theta_{S}$ with $\epsilon_{\infty}$. This is consistent with direct measurements of $\theta_{S}$ in Ref. [9] for a very similar PDMS 
system in the static limit—for which we remind that $\theta_{L}$ was also found independent of strain. Obviously, an important step for future work is to achieve fully self-consistent computations of the ridge dynamics that includes the strains induced by the Shuttleworth effect. This would, e.g., lead to a fully quantitative prediction of $\alpha\left(\theta_{S}\right)$.

Outlook.-Our study offers a general framework that establishes the laws of wetting on deformable soft solids, and which is consistent with all experimental observations. We have shown that equilibrium, in particular the lack of hysteresis, requires the equality of Shuttleworth coefficients $\gamma_{\mathrm{SL}}^{\prime}=\gamma_{\mathrm{SV}}^{\prime}$ for both the wet and dry solid. This sheds an unexpected light on coupling of physical chemistry, encoded in $\gamma(\epsilon)$, to the mechanics of wetting, and calls for a better understanding of the molecular origin of the Shuttleworth effect in cross-linked polymer networks $[25,26]$. This is of prime importance for the design and rheological characterisation of extremely soft materials, for which the interfacial effects dominate over bulk elasticity. From the perspective of wetting applications, we demonstrate that the Shuttleworth effect offers a new route to control the mobility of the contact line as illustrated here by stretching-enhanced spreading velocities.

This work was financially supported by the ANR grant Smart and ERC (the European Research Council) Consolidator Grant No. 616918.

[1] B. Roman and J. Bico, J. Phys. Condens. Matter 22, 493101 (2010).

[2] S. Mora, T. Phou, J.-M. Fromental, L. M. Pismen, and Y. Pomeau, Phys. Rev. Lett. 105, 214301 (2010).

[3] B. Andreotti and J. H. Snoeijer, Europhys. Lett. 113, 66001 (2016).

[4] R. W. Style, A. Jagota, C.-Y. Hui, and E. R. Dufresne, Annu. Rev. Condens. Matter Phys. 8, 99 (2017).

[5] J. Bico, É. Reyssat, and B. Roman, Annu. Rev. Fluid Mech. 50, 629 (2018).

[6] N. Naderman, C.-Y. Hui, and A. Jagota, Proc. Natl. Acad. Sci. U.S.A. 110, 10541 (2013).
[7] S. Mondal, M. Phukan, and A. Ghatak, Proc. Natl. Acad. Sci. U.S.A. 112, 12563 (2015).

[8] R. W. Style, R. Boltyanskiy, Y. Che, J. S. Wettlaufer, L. A. Wilen, and E. R. Dufresne, Phys. Rev. Lett. 110, 066103 (2013).

[9] Q. Xu, K. Jensen, R. Boltyanskiy, R. Sarfat, R. W. Style, and E. R. Dufresne, Nat. Commun. 8, 555 (2017).

[10] R. D. Schulman, M. Trejo, T. Salez, E. Raphael, and K. Dalnoki-Veress, Nat. Commun. 9, 982 (2018).

[11] R. W. Style and E. R. Dufresne, Soft Matter 8, 7177 (2012).

[12] A. Marchand, S. Das, J. H. Snoeijer, and B. Andreotti, Phys. Rev. Lett. 109, 236101 (2012).

[13] L. A. Lubbers, J. H. Weijs, L. Botto, S. Das, B. Andreotti, and J. H. Snoeijer, J. Fluid Mech. Rapids 747, R1 (2014).

[14] J. Dervaux and L. Limat, Proc. R. Soc. A 471, 20140813 (2015).

[15] Z. Cao and A. Dobrynin, Macromolecules 48, 443 (2015).

[16] A. Carré, J.-C. Gastel, and M. E. R. Shanahan, Nature (London) 379, 432 (1996).

[17] D. Long, A. Ajdari, and L. Leibler, Langmuir 12, 1675 (1996).

[18] T. Kajiya, A. Daerr, T. Narita, L. Royon, F. Lequeux, and L. Limat, Soft Matter 9, 454 (2013).

[19] J. Dupas, E. Verneuil, M. Van Landeghem, B. Bresson, L. Forny, M. Ramaioli, F. Lequeux, and L. Talini, Phys. Rev. Lett. 112, 188302 (2014).

[20] S. Karpitschka, S. Das, M. van Gorcum, H. Perrin, B. Andreotti, and J. H. Snoeijer, Nat. Commun. 6, 7891 (2015).

[21] R. Shuttleworth, Proc. Phys. Soc. London Sect. A 63, 444 (1950).

[22] A. Marchand, S. Das, J. H. Snoeijer, and B. Andreotti, Phys. Rev. Lett. 108, 094301 (2012).

[23] See Supplemental Material at http://link.aps.org/ supplemental/10.1103/PhysRevLett.121.068003 for technical details of the variational derivations and of the experiment.

[24] S. Das, A. Marchand, B. Andreotti, and J. H. Snoeijer, Phys. Fluids 23, 072006 (2011).

[25] H. Liang, Z. Cao, Z. Wang, and A. V. Dobrynin, ACS Macro Lett. 7, 116 (2018).

[26] Q. Xu, R. W. Style, and E. R. Dufresne, Soft Matter 14, 916 (2018).

[27] H. Perrin, R. Lhermerout, K. Davitt, E. Rolley, and B. Andreotti, Phys. Rev. Lett. 116, 184502 (2016). 